\title{
REMARKS ON SQUARE-INTEGRABLE BASIC COHOMOLOGY SPACES ON A FOLIATED RIEMANNIAN MANIFOLD
}

\author{
Dedicated to Professor T. Otsuki on his 60th birthday
}

\author{
By HARUO KitAHARA
}

B. L. Reinhart [7] showed that on compact foliated manifold with "bundlelike" metric, the cohomology of basic differential forms is isomorphic to the harmonic space of a certain semidefinite Laplacian. It is well known that the complex

$$
d^{\prime \prime}: o \longrightarrow \wedge^{0,0}(M) \longrightarrow \wedge^{0,1}(M) \longrightarrow \wedge^{0,2}(M) \longrightarrow \cdots
$$

is not elliptic. We shall define the completion $L_{2}^{0, s}(M)$ of compactly supported basic $(0, s)$-forms and discuss the squareintegrable basic cohomology spaces in complete case analogous to K. Okamoto and H. Ozeki [2] on a Hermitian manifold.

\section{$\S 1$. Definitions}

Let $M$ be an $n$ dimensional $C^{\infty}$ manifold which, topologically, is connected, orientable, paracompact Hausdorff space. We shall assume given on $M$ a foliation $E$ of codimension $q$, and we may find about each point a coordinate neighbourhood with coordinates $\left(x^{1}, \cdots, x^{p}, y^{1}, \cdots, y^{q}\right)(n=p+q)$ such that

(i) $\left|x^{2}\right| \leqq 1,\left|y^{\alpha}\right| \leqq 1$,

(ii) The integral manifolds of $E$ are given locally by $y^{1}=c^{1}, \cdots, y^{q}=c^{q}$ for constants $c^{\alpha}$ satisfying $\left|c^{\alpha}\right| \leqq 1$. (Here and hereafter, Latin indices run from 1 to $p$, and Greek indices from 1 to q.)

Such a coordinate neighbourhood will be called flat, while each of slices given by a set of equations $y^{\alpha}=c^{\alpha}$ will be called a plaque. If $U$ is a flat neighbourhood, the quotient space of $U$ by its plaques will be called the local base and be denoted by $U_{y}$.

We may assume that there exist in $U$ differential forms $w^{2}$ and vectors $v_{\alpha}$ such that

(i) $\left\{\partial / \partial x^{i}\right\}$ forms the base for the space of cross-sections of $E$ in $U$ at each point,

Received December 17, 1977 
(ii) $\left\{w^{1}, \cdots, w^{p}, d y^{1}, \cdots, d y^{q}\right\}$ and $\left\{\partial / \partial x^{1}, \cdots, \partial / \partial x^{p}, v_{1}, \cdots, v_{q}\right\}$ are dual bases for the cotangent and tangent spaces at each point of $U$ respectively. Hence, $w^{2}=d x^{2}+\sum_{\alpha} a_{\alpha}^{2} d y^{\alpha}$ and $v_{\alpha}=\partial / \partial y^{\alpha}+\sum_{\imath} b_{\alpha}^{2} \partial / \partial x^{2}$.

Throughout this note, all local expressions for differential forms and vectors will be taken with respect to these bases.

\section{$\S 2$. Square-integrable basic cohomology spaces}

On a foliated manifold we may have the decomposition of differential forms into components in following way: Any $C^{\infty}-m$-form $\phi$ may be expressed locally as

$$
\sum_{\alpha_{1}^{2}<\cdots<\alpha_{s}^{2} r} \sum_{r+s=m} \phi_{i_{1} \cdots \imath_{r} \alpha_{1} \cdots \alpha_{s}}(x, y) w^{\imath_{1}} \wedge \cdots \wedge w^{\imath_{r}} \wedge d y^{\alpha_{1}} \wedge \cdots \wedge d y^{\alpha_{s}} .
$$

We then define $\Pi_{r, s} \phi$ to be the sum of all these terms with a fixed $r$ and $s$. Since under change of flat coordinate system, $\left\{\left\{d y^{\alpha}\right\}\right\}$ goes into $\left\{\left\{d y^{* \alpha}\right\}\right\}$ and $\left\{\left\{w^{i}\right\}\right\}$ goes into $\left\{\left\{w^{* i}\right\}\right\}$, this operator $\Pi_{r, s}$ is independent of the choice of coordinate system. Here by $\{\{\cdot\}\}$ we mean the vector space generated by the set $\{\cdot\} . \Pi_{r, s} \phi$ is called the component of type $(r, s)$ of $\phi$. The type decomposition of forms induces a type decomposition of the exterior derivative $d$ by the rule $\left(\Pi_{t, u} d\right) \phi=\sum_{r, s} \Pi_{r+t, s+u} d \Pi_{r, s} \phi$. Let $\Pi_{1,0} d=d^{\prime}$ and $\Pi_{0,1} d=d^{\prime \prime}$. In general, there will be a component $\Pi_{-1,2} d$; since we are interested only in forms of type $(0, s)$, we shall not introduce a notion for this component.

Proposition 2.1. (cf. [7]) If $\phi$ is of type $(0, s)$, then $d \phi=d^{\prime} \phi+d^{\prime \prime} \phi$. Moreover, $d^{\prime} \phi=0$ if and only if $\phi$ depends only upon $y$, in the sense that locally

$$
\phi=\sum \phi_{\alpha_{1} \cdots \alpha_{s}}(y) d y^{\alpha_{1}} \wedge \cdots \wedge d y^{\alpha_{s}} .
$$

Definition 2.1. A form of type $(0, s)$ which is annihilated by $d^{\prime}$ will be called a basic form.

Definition 2.2. A riemannian metric is bundle-like if and only if it is representable in each flat neighbourhood by an expression of the form

$$
d s^{2}=\sum g_{\imath j}(x, y) w^{2} w^{\jmath}+\sum g_{\alpha \beta}(y) d y^{\alpha} d y^{\beta} .
$$

Hereafter, we assume that the riemannian metric on $M$ is bundle-like and all leaves are compact.

Remark. In this paragraph, we may replace "all leaves are compact" by "the volume of $M$ is finite", then the compact support of the basic form is replaced by the tranversally compact support, i. e. $\phi_{\alpha_{1} \cdots \alpha_{s}}(y) d y^{\alpha_{1}} \wedge \cdots \wedge d y^{\alpha_{s}}$ has compact support in variable $y^{\prime} s$ in local expression, and, for example, we may consider the torus foliated by a family of irrational spirals.

Let $\wedge^{0, s}(M)$ be the space of all $C^{\infty}$-basic form of type $(0, s)$ and $\wedge_{0}^{0, s}(M)$ the subspace of $\wedge^{0, s}(M)$ composed of forms with compact supports. Restricted to 
$\wedge^{0, *}(M)=\sum_{s=0}^{\infty} \wedge^{0, s}(M), d^{\prime \prime 2}=d^{2}=0$, so we may consider the cohomology of $\wedge^{0, *}(M)$ and $d^{\prime \prime}$. (This is called the base-like cohomology by B. L. Reinhart [7].)

B. L. Reinhart [7] introduces the ${ }^{* \prime \prime}$-operation on $\wedge^{0, s}(M)$, and defined by

$$
\begin{aligned}
& \text { *" } \phi=\sum_{\substack{\alpha_{1} \leq:<\alpha_{s} \\
\beta_{1}<\beta_{q-s}}} \operatorname{sgn}\left(\begin{array}{c}
1 \cdots \cdots \cdots \cdots \cdots \cdots \cdots \cdot \\
\alpha_{1} \cdots \alpha_{s} \beta_{1} \cdots \beta_{q-s}
\end{array}\right)\left(\operatorname{det}\left(g_{\alpha \beta}\right)\right)^{1 / 2} \\
& g^{\alpha_{1} \nu_{1}} \cdots g^{\alpha_{s} \nu_{s}} \phi_{\nu_{1} \cdots \nu_{s}} d y^{\beta_{1}} \wedge \cdots \wedge d y^{\beta_{q-s}} .
\end{aligned}
$$

According to B. L. Reinhart [7], we may define a riemannian metric on $\wedge^{0, s}(M)$ by

$$
\langle\phi, \phi\rangle=\phi \wedge^{* \prime \prime} \phi \wedge d x^{1} \wedge \cdots \wedge d x^{p},
$$

and obtain a pre-Hilbertian metric on $\wedge_{o}^{0, s}(M)$ by

$$
\begin{aligned}
(\phi, \phi) & =\int_{M}\langle\phi, \phi\rangle \\
& =\int_{M} \phi \wedge^{* \prime \prime} \phi \wedge d x^{1} \wedge \cdots \wedge d x^{p} .
\end{aligned}
$$

The differential operator $d^{\prime \prime}$ maps $\wedge^{0, s}(M)$ into $\wedge^{0, s+1}(M)$. We define $\delta^{\prime \prime}$ : $\wedge^{0, s}(M) \rightarrow \wedge^{0, s-1}(M)$ by

Then we have

$$
\delta^{\prime \prime} \phi=(-1)^{q s+q+1 * \prime \prime} d^{\prime \prime * \prime \prime} \phi .
$$

$$
\left(d^{\prime \prime} \phi, \phi\right)=\left(\phi, \delta^{\prime \prime} \phi\right)
$$

for $\left.\phi \in \wedge_{o}^{0, s} M\right), \phi \in \wedge_{o}^{0, s+1}(M)$.

Let $L_{2}^{0, s}(M)$ be the completion of $\wedge_{0}^{0, s}(M)$ with respect to the inner product (, ). We will denote by $\partial$ the restriction of $d^{\prime \prime}$ to $\wedge_{o}^{0, s}(M)$ and by $\theta$ the restriction of $\delta^{\prime \prime}$ to $\wedge_{0}^{0, s}(M)$. Define

$$
\bar{\partial}=(\theta)^{*} \text { and } \bar{\theta}=(\partial)^{*}
$$

where ()$^{*}$ denotes the adjoint operator of () with respect to the inner product ( ,). Then $\bar{\partial}$ (resp. $\bar{\theta}$ ) is a closed, densely defined operator of $L_{2}^{0, s}(M)$ into $L_{2}^{0, s+1}(M)$ (resp. $\left.L_{2}^{0, s-1}(M)\right)$. Let $D_{\bar{\partial}}^{0, s}\left(\right.$ resp. $\left.D_{\bar{\theta}}^{0, s}\right)$ be the domain of the operator $\bar{\partial}$ (resp. $\bar{\theta}$ ) in $L_{2}^{0, s}(M)$. We put

$$
\begin{aligned}
& Z_{\bar{\partial}}^{0, s}(M)=\left\{\phi \in D_{\bar{\partial}}^{0, s} \mid \bar{\partial} \phi=0\right\} \\
& Z_{\bar{\theta}}^{0, s}(M)=\left\{\phi \in D_{\bar{\theta}}^{0, s} \mid \bar{\theta} \phi=0\right\} .
\end{aligned}
$$

Since $\bar{\partial}$ and $\bar{\theta}$ are closed operators, $Z_{\bar{\partial}}^{0, s}(M)$ and $Z_{\bar{\theta}}^{0, s}(M)$ are closed in $L_{2}^{0, s}(M)$. Let $B_{\bar{\partial}}^{0, s}(M)$ and $B_{\bar{\theta}}^{0, s}(M)$ be the closure of $\bar{\partial}\left(D_{\bar{\partial}}^{0, s-1}\right)$ and $\bar{\theta}\left(D_{\bar{\theta}}^{0, s+1}\right)$ respectively.

Definition 2.3. $H_{2}^{0, s}(M)=Z_{\overline{\hat{o}}}^{0, s}(M) \ominus B_{\overline{\bar{o}}}^{0, s}(M)$ is the square-integrable basic cohomology spaces, where $\ominus$ denotes the orthogonal complement of $B_{\bar{\partial}}^{0, s}(M)$.

It is easy to see that 


$$
H_{2}^{0, s}(M)=Z_{\bar{\partial}}^{0, s}(M) \cap Z_{\bar{\theta}}^{0, s}(M) .
$$

Since $Z_{\bar{\partial}}^{0, s}(M)$ and $Z_{\bar{\theta}}^{0, s}(M)$ are closed in $L_{2}^{0, s}(M), H_{2}^{0, s}(M)$ has canonically the structure of a Hilbert space.

THEOREM 2.1. (The orthogonal decomposition theorem)

$$
L_{2}^{0, s}(M)=H_{2}^{0, s}(M) \oplus B_{\bar{\partial}}^{0, s}(M) \oplus B_{\bar{\theta}}^{0, s}(M) .
$$

Proof is analogous to L. Hörmander [3], in fact, we have only to notice that $B_{\bar{\partial}}^{0, s}(M)$ and $B_{\bar{\theta}}^{0, s}(M)$ are mutually orthgonal and $B_{\bar{\partial}}^{0, s}(M)^{\perp} \cap B_{\bar{\theta}}^{0, s}(M)^{\perp}=H_{2}^{0, s}(M)$, where $\perp$ denotes the orthogonal complement in $L_{2}^{0, s}(M)$.

Then we have the Dolbeault-Serre type theorem.

THEOREM 2.2. If the bundle-like metruc on $M$ is complete, then,

$$
H_{2}^{0, s}(M)=H_{2}^{0, q-s}(M) \quad \text { (isomorphic as Hilbert space). }
$$

In fact, we have only to notice that the following diagram is commutative.

$$
\begin{gathered}
\wedge_{0}^{0, s}(M) \stackrel{* \prime \prime}{\longrightarrow} \wedge_{0}^{0, q-s}(M) \\
\bar{\theta} \downarrow \uparrow \bar{\partial}(-1)^{s * \prime \prime} \quad \bar{\partial} \downarrow \uparrow \uparrow \bar{\theta} \\
\wedge_{0}^{0, s-1}(M) \stackrel{{ }^{0, q-s+1}(M) .}{\longrightarrow} \wedge_{0}^{0, q-1}(M)
\end{gathered}
$$

Corollary 2.1. (cf. [7]) If $\operatorname{dim} H_{2}^{0, s}(M)$ is finite, then $\operatorname{dim} H_{2}^{0, s}(M)=\operatorname{dim}$ $H_{2}^{0, q-s}(M)$.

\section{$\S 3$. Harmonic forms in complete bundle-like metric}

Hereafter, we assume that the bundle-like metric is complete and all leaves are compact.

Proposition 3.1.

$$
\begin{aligned}
& N_{d^{\prime}}^{0, s}(M) \cap L_{2}^{0, s}(M) \subset Z_{\bar{\partial}}^{0, s}(M) \\
& N_{\delta^{*}}^{0, s}(M) \cap L_{2}^{0, s}(M) \subset Z_{\bar{\theta}}^{0, s}(M),
\end{aligned}
$$

where $N_{d^{\prime \prime}}^{0, s}(M)=\left\{\phi \in \wedge^{0, s}(M) \mid d^{\prime \prime} \phi=0\right\}$ and $N_{\delta^{\prime \prime}}^{0, s}(M)=\left\{\phi \in \wedge^{0, s}(M) \mid \delta^{\prime \prime} \phi=0\right\}$.

In order to prove this proposition, we need some facts analogous to $\mathrm{A}$. Andreotti and E. Vesentini [1].

We consider a differentiable function $\mu$ on $\boldsymbol{R}$ (the reals) satisfying

(i) $0 \leqq \mu \leqq 1$ on $\boldsymbol{R}$,

(ii) $\mu(t)=1$ for $t \leqq 1$,

(iii) $\mu(t)=0$ for $t \geqq 2$.

It is known that a geodesic orthogonal to a leaf is orthogonal to all leaves (cf. B. L. Reinhart [6]). We fix a point $o$ in $M$, and for each point $p$ in $M$, we 
denote by $\rho(p)$ the distance between leaves through $o$ and $p$. Then we set

$$
w_{k}(p)=\mu(\rho(p) / k) \quad \text { for } \quad k=1,2,3, \cdots .
$$

LEMMA 3.1. Under the above notations, there exists a positive number $A$, depending only on $\mu$, such that

(i) $\left\|d^{\prime \prime} w_{k} \wedge \phi\right\|^{2}<\frac{n A^{2}}{k^{2}}\|\phi\|^{2}$

(ii) $\left\|d^{\prime \prime} w_{k} \wedge^{* \prime \prime} \phi\right\|^{2}<\frac{n A^{2}}{k^{2}}\|\phi\|^{2}$

for all $\phi \in \wedge^{0, s}(M)$, where $\|\phi\|^{2}=(\phi, \phi)$.

In order to prove this lemma, we have to notice that the function $\rho(p)$ is a locally Lipschitz function and, at points where the derivatives exist, it holds

Then we have

$$
\sum g^{\alpha \beta} v_{\alpha}(\rho) v_{\beta}(\rho)<n
$$

$$
\left|d^{\prime \prime} w_{k}\right|^{2}=\sum g^{\alpha \beta} v_{\alpha}\left(w_{k}\right) v_{\beta}\left(w_{k}\right)<\frac{n A^{2}}{k^{2}}
$$

where $A$ is a positive number depending only on $\sup \left|\frac{d \mu}{d t}\right|$.

We remark that $d^{\prime} w_{k}=0$ and $w_{k} \phi$ has compact support for each $\phi \in \wedge^{0, s}(M)$. Then we have that $w_{k} \phi \in D_{\bar{\partial}}^{0, s} \cap D_{\bar{\theta}}^{0, s}$ for $\phi \in \wedge^{0, s}(M)$, and that

$$
\begin{aligned}
& \bar{\partial}\left(w_{k} \phi\right)=d^{\prime \prime}\left(w_{k} \phi\right) \\
& \bar{\theta}\left(w_{k} \phi\right)=\delta^{\prime \prime}\left(w_{k} \phi\right) .
\end{aligned}
$$

Now we prove Proposition 3.1. Let $\phi$ be in $N_{d^{n}}^{0, s} \cap L_{2}^{0, s}(M)$. By the above remarks

$$
\begin{aligned}
\bar{\partial}\left(w_{k} \phi\right) & =d^{\prime \prime}\left(w_{k} \phi\right) \\
& =d^{\prime \prime} w_{k} \wedge \phi+w_{k} d^{\prime \prime} \phi \\
& =d^{\prime \prime} w_{k} \wedge \phi .
\end{aligned}
$$

Hence, by Lemma 3.1, we have

$$
\left\|\bar{\partial}\left(w_{k} \phi\right)\right\|^{2}<\frac{n A^{2}}{k^{2}}\|\phi\|^{2} .
$$

Putting $\phi_{k}=w_{k} \phi$, we we have

$$
\bar{\partial} \phi_{k} \longrightarrow 0 \quad(k \longrightarrow \infty) \quad \text { (strong). }
$$

On the other hand, $\phi_{k} \rightarrow \phi(k \rightarrow \infty)$ (strong). Since $\bar{\partial}$ is a closed operator, we see that $\phi$ is in $D_{\bar{\partial}}^{0, s}$ and $\bar{\partial} \phi=0$. This proves $\phi \in Z_{\bar{\partial}}^{0, s}(M)$. In the same way, we have $N_{\delta^{\prime \prime}}^{0, s} \cap L_{2}^{0, s}(M) \subset Z_{\bar{\theta}}^{0, s}(M)$. This proves Proposition 3.1. 
Definition 3.1. The Laplacian acting on $\wedge^{0 * *}(M)$ is defined by

$$
\square=-\left(d^{\prime \prime} \delta^{\prime \prime}+\delta^{\prime \prime} d^{\prime \prime}\right) \text {. }
$$

For any $\phi \in L_{2}^{0, s}(M) \cap \wedge^{0, s}(M)$, we have

$$
\left(d^{\prime \prime} \phi, d^{\prime \prime} \alpha\right)_{B(k)}+\left(\delta^{\prime \prime} \phi, \delta^{\prime \prime} \alpha\right)_{B(k)}=(-\square \phi, \alpha)_{B(k)}
$$

for all $\alpha \in \wedge_{B(k)}^{0, s}(M)$, where $\wedge_{B(k)}^{0, s}(M)$ is the space of all forms of type $(0, s)$ with compact support contained in $B(k)$ and $B(k)$ is an open tube of radius $k$ of the leaf through the fixed point $o$ in $M$. For $\alpha=w_{k}^{2} \phi$, we have

$$
\begin{aligned}
& d^{\prime \prime} \alpha=w_{k}^{2} d^{\prime \prime} \phi+2 w_{k} d^{\prime \prime} w_{k} \wedge \phi \\
& \delta^{\prime \prime} \alpha=w_{k}^{2} \delta^{\prime \prime} \phi+(-1)^{q s+q+1} * \prime \prime\left(2 w_{k} d^{\prime \prime} w_{k} \wedge^{* \prime \prime} \phi\right) .
\end{aligned}
$$

Substituting in (3.1), we have

$$
\begin{aligned}
& \left\|w_{k} d^{\prime \prime} \phi\right\|_{B(k)}^{2}+\left\|w_{k} \delta^{\prime \prime} \phi\right\|_{B(k)}^{2} \\
\leqq & \left|\left(\square \phi, w_{k}^{2} \phi\right)_{B(k)}\right|+\left|\left(d^{\prime \prime} \phi, 2 w_{k} d^{\prime \prime} w_{k} \wedge \phi\right)_{B(k)}\right| \\
& +\left|\left(\delta^{\prime \prime} \phi, * \prime \prime\left(2 w_{k} d^{\prime \prime} w_{k} \wedge^{* \prime \prime} \phi\right)\right)_{B(k)}\right| .
\end{aligned}
$$

On the other hand, the Schwartz inequality gives the following

$$
\begin{aligned}
& \left|\left(d^{\prime \prime} \phi, 2 w_{k} d^{\prime \prime} w_{k} \wedge \phi\right)_{B(k)}\right| \leqq \frac{1}{2}\left(\left\|w_{k} d^{\prime \prime} \phi\right\|_{B(k)}^{2}+4\left\|d^{\prime \prime} w_{k} \wedge \phi\right\|_{B(k)}^{2}\right) \\
& \left|\left(\delta^{\prime \prime} \phi,{ }^{* \prime \prime}\left(2 w_{k} d^{\prime \prime} w_{k} \wedge^{* \prime \prime} \phi\right)\right)_{B(k)}\right| \leqq \frac{1}{2}\left(\left\|w_{k} \delta^{\prime \prime} \phi\right\|_{B(k)}^{2}+4\left\|d^{\prime \prime} w_{k} \wedge^{* \prime \prime} \phi\right\|_{B(k)}^{2}\right)
\end{aligned}
$$

and

$$
\left|\left(\square \phi, w_{k}^{2} \phi\right)_{B(k)}\right| \leqq \frac{1}{2}\left(\frac{1}{\sigma}\left\|w_{k} \phi\right\|_{B(k)}^{2}+\sigma\|\square \phi\|_{B(k)}^{2}\right)
$$

for every $\sigma>0$.

Substituting in (3.2),

$$
\begin{aligned}
\left\|w_{k} d^{\prime \prime} \phi\right\|_{B(k)}^{2} & +\left\|w_{k} \delta^{\prime \prime} \phi\right\|_{B(k)}^{2} \\
& <\sigma\|\square \phi\|_{B(k)}^{2}+\left(\frac{1}{\sigma}+\frac{8 n A^{2}}{k^{2}}\right)\|\phi\|_{B(k)}^{2} .
\end{aligned}
$$

Letting $k \rightarrow \infty$, we have

$$
\left\|d^{\prime \prime} \phi\right\|^{2}+\left\|\delta^{\prime \prime} \phi\right\|^{2}<\sigma\|\square \phi\|^{2}+\frac{1}{\sigma}\|\phi\|^{2}
$$

for every $\sigma>0$. In particular, setting $\square \phi=0$ and letting $\sigma \rightarrow \infty$, we have

LEMMA 3.2. Let the bundle-like metric on $M$ be complete and all leaves be compact. If $\phi \in L_{2}^{0, s}(M) \cap \wedge^{0, s}(M)$ such that $\square \phi=0$, then $d^{\prime \prime} \phi=0$ and $\delta^{\prime \prime} \phi=0$, i. e. $\phi \in N_{d^{\prime}}^{0, s}(M) \cap N_{\delta^{\prime}}^{0, s}(M)$. 
From Proposition 3.1 and Lemma 3.2, we have the following theorem.

THEOREM 3.1. Let the bundle-like metric on $M$ be complete and all leaves be compact. If $\phi \in L_{2}^{0, s}(M) \cap \wedge^{0, s}(M)$ such that $\square \phi=0$, then $\phi \in H_{2}^{0, s}(M)$.

Remark. The bundle-like metric can be deformed to a continuous complete metric if all leaves are compact, but the deformed metric can not be $C^{\infty}$-metric (cf. $[4,5])$.

Remark. It is well known by I. Vaisman [8] that for a compact oriented foliated riemannian manifold $M$, the space $\mathcal{H}^{r, s}(M)$ of foliated harmonic forms is a subspace of the de Rham cohomology space $H^{r, s}(M)$.

\section{BIBLIOGRAPHY}

[1] A. Andreotti and E. Vesentini, Carleman estimates for the Laplace-Beltrami equation on complex manifolds, Inst. Hautes Etudes Sci. Publ. Math. 25 (1965), 313-362.

[2] K. Окамото AND H. OzeKI, On square-integrable $\bar{\partial}$-cohomology spaces attached to hermitian symmetric spaces, Osaka J. Math 4 (1967), 95-110.

[3] L. Hörmander, $L^{2}$-estimates and existence theorems for the $\bar{\partial}$-operator, Acta Math. 113 (1965), 89-152.

[4] H. Kitahara, The existence of complete bundle-like metrics, Ann. Sci. Kanazawa Univ. 9 (1972), 37-40.

[5] H. Kitahara, The existence of complete bundle-like metrics, II, Ann. Sci. Kanazawa Univ. 10 (1973), 51-54.

[6] B.L. ReInHART, Foliated manifolds with bundle-like metrics, Ann. of Math. 69 (1959), 119-132.

[7] B.L. Reinhart, Harmonic integrals on foliated manifolds, Amer. J. Math. 81 (1959), 529-536.

[8] I. Vaisman, Variétés rıemanniennes feuilletées, Czechoslovak Math. J. 21 (1971), 46-75.

Department of Mathematics

College of Liberal Arts

Kanazawa University 